\title{
Effect algebras with the maximality property
}

\author{
Josef Tkadlec \\ Department of Mathematics, Faculty of Electrical Engineering, \\ Czech Technical University, 16627 Praha, Czech Republic, \\ tkadlec@fel.cvut.cz
}

\begin{abstract}
The maximality property was introduced in [7] in orthomodular posets as a common generalization of orthomodular lattices and orthocomplete orthomodular posets. We show that various conditions used in the theory of effect algebras are stronger than the maximality property, clear up the connections between them and show some consequences of these conditions. In particular, we prove that a Jauch-Piron effect algebra with a countable unital set of states is an orthomodular lattice and that a unital set of Jauch-Piron states on an effect algebra with the maximality property is strongly order determining.
\end{abstract}

\section{Basic notions}

Effect algebras as generalizations of orthomodular posets (quantum logics) are studied in the axiomatics of quantum systems - see, e.g., [1, 2].

1.1. Definition. An effect algebra is an algebraic structure $(E, \oplus, \mathbf{0}, \mathbf{1})$ such that $E$ is a set, $\mathbf{0}$ and $\mathbf{1}$ are different elements of $E$ and $\oplus$ is a partial binary operation on $E$ such that for every $a, b, c \in E$ the following conditions hold:

(1) $a \oplus b=b \oplus a$ if $a \oplus b$ exists,

(2) $(a \oplus b) \oplus c=a \oplus(b \oplus c)$ if $(a \oplus b) \oplus c$ exists,

(3) there is a unique $a^{\prime} \in E$ such that $a \oplus a^{\prime}=\mathbf{1}$ (orthosupplement),

(4) $a=\mathbf{0}$ whenever $a \oplus \mathbf{1}$ is defined.

For simplicity, we use the notation $E$ for an effect algebra. A partial ordering on an effect algebra $E$ is defined by $a \leq b$ iff there is a $c \in E$ such that $b=a \oplus c$. Such an element $c$ is unique (if it exists) and is denoted by $b \ominus a . \mathbf{0}(\mathbf{1}$, resp.) is the least (the greatest, resp.) element of $E$ with respect to this partial ordering. For every $a, b \in E, a^{\prime \prime}=a$ and $b^{\prime} \leq a^{\prime}$ whenever $a \leq b$. It can be shown that $a \oplus \mathbf{0}=a$ for every $a \in E$ and that a cancellation law is valid: for every $a, b, c \in E$ with $a \oplus b \leq a \oplus c$ we have $b \leq c$. An orthogonality relation on $E$ is defined by $a \perp b$ iff $a \oplus b$ exists (iff $a \leq b^{\prime}$ ). (See, e.g., [1, 2].)

For $a \leq b$ we denote $[a, b]=\{c \in E: a \leq c \leq b\}$. A chain in $E$ is a nonempty linearly (totally) ordered subset of $E$.

Obviously, if $a \perp b$ and $a \vee b$ exist in an effect algebra, then $a \vee b \leq a \oplus b$. The reverse inequality need not be true (it holds in orthomodular posets).

1.2. Definition. Let $E$ be an effect algebra. An element $a \in E$ is principal if $b \oplus c \leq a$ for every $b, c \in E$ such that $b, c \leq a$ and $b \perp c$.

1.3. Definition. An orthoalgebra is an effect algebra $E$ in which, for every $a \in E$, $a=\mathbf{0}$ whenever $a \oplus a$ is defined.

An orthomodular poset is an effect algebra in which every element is principal.

An orthomodular lattice is an orthomodular poset that is a lattice. 
Every orthomodular poset is an orthoalgebra. Indeed, if $a \oplus a$ is defined then $a \oplus a \leq a=a \oplus \mathbf{0}$ and, accordig to the cancellation law, $a \leq \mathbf{0}$ and therefore $a=\mathbf{0}$.

Orthoalgebras are characterized by the following conditions: the orthosupplementation is an orthocomplementation (i.e., $a \vee a^{\prime}=\mathbf{1}$ for every $a$ ) or $a \oplus b$ is a minimal upper bound of $a, b$ for every $a, b$. Orthomodular posets are characterized as effect algebras such that $a \oplus b=a \vee b$ for every orthogonal pair $a, b$. (See [2, 3].) Let us remark that an orthomodular poset is usually defined as a bounded partially ordered set with an orthocomplementation in which the orthomodular law is valid.

Let us present a special class of orthomodular posets that we will use in some examples.

1.4. Proposition. Let $X \neq \emptyset, E \subset \exp X$ be nonempty such that the following conditions are fulfilled:

(1) $X \backslash A \in E$ whenever $A \in E$,

(2) $A \cup B \in E$ whenever $A, B \in E$ are disjoint.

Then $(E, \oplus, \emptyset, X)$ with $A \oplus B=A \cup B$ for disjoint $A, B \in E$ is an orthomodular poset such that the orthosupplement is the set-theoretic complement and the partial ordering is the inclusion.

Proof. Since $E$ is nonempty, there is an element $A \in E$. According to the condition (1), $X \backslash A \in E$. According to the condition (2), $X=A \cup(X \backslash A) \in E$ and, according to the condition (1), $\emptyset=X \backslash X \in E$. It is easy to see that the axioms of an effect algebra are fulfilled, that the orthosupplement is the set-theoretic complement, that the partial ordering is the inclusion and that every element of $E$ is principal.

1.5. Definition. An orthomodular poset of the form of Proposition 1.4 is called concrete.

Let us present two important notions we will use in the sequel.

1.6. Definition. A system $\left(a_{i}\right)_{i \in I}$ of (not necessarilly distinct) elements of an effect algebra $E$ is orthogonal if $\bigoplus_{i \in F} a_{i}$ is defined for every finite set $F \subset I$.

An effect algebra $E$ is orthocomplete if for every orthogonal system $\left(a_{i}\right)_{i \in I}$ of elements of $E$ the supremum $\bigvee\left\{\bigoplus_{i \in F} a_{i}: F \subset I\right.$ is finite $\}$ exists.

1.7. Definition. An effect algebra $E$ has the maximality property if $[\mathbf{0}, a] \cap[\mathbf{0}, b]$ has a maximal element for every $a, b \in E$.

Obviously, every finite effect algebra has the maximality property and every lattice effect algebra has the maximality property $-a \wedge b$ is a maximal (even the greatest) element of $[\mathbf{0}, a] \cap[\mathbf{0}, b]$ for every $a, b$.

\section{States}

2.1. Definition. Let $E$ be an effect algebra. A state $s$ on $E$ is a mapping $s$ : $E \rightarrow[0,1]$ such that: 
(1) $s(\mathbf{1})=1$

(2) $s(a \oplus b)=s(a)+s(b)$ whenever $a \oplus b$ is defined.

A set $S$ of states on $E$ is unital, if for every $a \in E \backslash\{\mathbf{0}\}$ there is a state $s \in S$ such that $s(a)=1$.

A set $S$ of states on $E$ is strongly order determining, if for every $a, b \in E$ with $a \not \leq b$ there is a state $s \in S$ such that $s(a)=1>s(b)$.

Obviously, for every state $s$ we have $s(\mathbf{0})=0, s\left(a^{\prime}\right)=1-s(a)$ for every $a \in E$, $s(a) \leq s(b)$ for every $a, b \in E$ with $a \leq b$.

There are special two-valued states on concrete orthomodular posets (it is easy to verify that they are indeed states):

2.2. Definition. Let $E \subset \exp X$ be a concrete orthomodular poset, $x \in X$. The state $s_{x}$ on $E$ defined by

$$
s_{x}(A)=\left\{\begin{array}{ll}
0, & x \notin A, \\
1, & x \in A,
\end{array} \quad A \in E,\right.
$$

is called carried by the point $x$.

It is easy to see that for concrete orthomodular posets the set of states carried by points is strongly order determining.

2.3. Lemma. Every strongly order determining set of states on an effect algebra is unital.

Proof. Let $S$ be a strongly order determining set of states on an effect algebra $E$, $a$ be a nonzero element of $E$. Then $a \not \leq \mathbf{0}$ and therefore there is a state $s \in S$ such that $s(a)=1>s(\mathbf{0})$.

Let us present two observations describing the impact of a sufficiently large state spaces to the properties of the algebraic structure.

2.4. Proposition. Every effect algebra with a unital set of states is an orthoalgebra.

Proof. Let $E$ be an effect algebra with a unital set $S$ of states. Let $a \in E$ be such that $a \oplus a$ is defined. Then $1 \geq s(a \oplus a)=2 s(a)$ and therefore $s(a)<\frac{1}{2}$ for every state $s \in S$. Since $S$ is unital, we obtain that $a=\mathbf{0}$.

2.5. Proposition. Every effect algebra with a strongly order determining set of states is an orthomodular poset.

Proof. Let $E$ be an effect algebra with a strongly order determining set $S$ of states. Let us prove that every element of $E$ is principal. Let $a, b, c \in E$ such that $b, c \leq a$ and $b \perp c$. Then for every state $s \in S$ with $s\left(a^{\prime}\right)=1$ we consecutively obtain: $0=s(a)=s(b)=s(c)=s(b \oplus c), s\left((b \oplus c)^{\prime}\right)=1$. Since the set $S$ is strongly order determining, we obtain that $a^{\prime} \leq(b \oplus c)^{\prime}$ and therefore $b \oplus c \leq a$. 


\section{Jauch-Pironness}

3.1. Definition. Let $E$ be an effect algebra. A state $s$ on $E$ is Jauch-Piron if for every $a, b \in E$ with $s(a)=s(b)=1$ there is a $c \in E$ such that $c \leq a, b$ and $s(c)=1$.

An effect algebra is Jauch-Piron if every state on it is Jauch-Piron.

The following statement was proved in [6, Proposition 2.6], we will generalize it later (Theorem 3.7).

3.2. Lemma. Every Jauch-Piron effect algebra with a countable unital set of states has the maximality property.

Proof. Let $E$ be a Jauch-Piron effect algebra with a countable unital set $S$ of states. Let $a, b \in E$. If $[\mathbf{0}, a] \cap[\mathbf{0}, b]=\{\mathbf{0}\}$ then $\mathbf{0}$ is a maximal element of $[\mathbf{0}, a] \cap[\mathbf{0}, b]$. Let us suppose that $[\mathbf{0}, a] \cap[\mathbf{0}, b] \neq\{\mathbf{0}\}$. Then there is an element $c \in[\mathbf{0}, a] \cap[\mathbf{0}, b] \backslash\{\mathbf{0}\}$ and, since the set $S$ is unital, there is a state $s \in S$ such that $s(c)=1$. Hence $s(a)=s(b)=1$ and the set $S_{a, b}=\{s \in S: s(a)=s(b)=1\}$ is nonempty and countable. Let $s_{0}$ be a $\sigma$-convex combination (with nonzero coefficients) of all states from $S_{a, b}$. Then $s_{0}(a)=s_{0}(b)=1$. Since the state $s_{0}$ is Jauch-Piron, there is an element $c \in[\mathbf{0}, a] \cap[\mathbf{0}, b]$ such that $s_{0}(c)=1$. It remains to prove that $c$ is a maximal element of $[\mathbf{0}, a] \cap[\mathbf{0}, b]$. Indeed, if $d \in[\mathbf{0}, a] \cap[\mathbf{0}, b]$ with $d \geq c$ then $e=d \ominus c \in[\mathbf{0}, a] \cap[\mathbf{0}, b]$ and $e \perp c$. Hence $s_{0}(e)=0$ and therefore there is no state $s \in S$ such that $s(e)=1$. Due to the unitality of $S, e=0$ and therefore $d=c$.

3.3. Proposition. Every effect algebra with the maximality property and with a unital set of Jauch-Piron states is an orthomodular poset.

Proof. Let $E$ be an effect algebra with the maximality property and with a unital set $S$ of Jauch-Piron states. Let us suppose that $E$ is not an orthomodular poset and seek a contradiction. There are elements $a, b, c \in E$ such that $b, c \leq a, b \perp c$ and $b \oplus c \not \leq a$. Let us denote $d=b \oplus c$. Since $E$ has the maximality property, there is a maximal element $e$ in $\left[\mathbf{0}, a^{\prime}\right] \cap\left[\mathbf{0}, d^{\prime}\right]$. Since $d \not \leq a$, we obtain that $a^{\prime} \not \leq d^{\prime}$ and therefore $e<a^{\prime}$ and $a^{\prime} \ominus e \neq \mathbf{0}$. Since the set $S$ is unital, there is a state $s \in S$ such that $s\left(a^{\prime} \ominus e\right)=1$. Hence $s\left(a^{\prime}\right)=1,0=s(e)=s(a)=s(b)=s(c)=s(d), s\left(d^{\prime}\right)=1$, $s\left(d^{\prime} \ominus e\right)=1$. Since the state $s$ is Jauch-Piron, there is an element $f \in E$ such that $f \leq\left(a^{\prime} \ominus e\right),\left(d^{\prime} \ominus e\right)$ and $s(f)=1$. Hence $f \neq \mathbf{0}$ and $e<e \oplus f \leq a^{\prime}, d^{\prime}$ - this contradicts to the maximality of $e$.

Let us remark that there are effect algebras with the maximality property that are not orthoalgebras - e.g., the 3-chain $C_{3}=\{\mathbf{0}, a, \mathbf{1}\}$ with $a \oplus a=\mathbf{1}$ and $x \oplus$ $\mathbf{0}=x$ for every $x \in C_{3}$. It seems to be an open question whether the assumption of the maximality property in Proposition 3.3 might be omitted (it is not a consequence of the existence of a countable unital set of Jauch-Piron states - see Example 3.8.) Proposition 3.3 cannot be improved to orthomodular lattices - see Example 3.5 ( $\left\{\frac{1}{2}\left(s_{x}+s_{y}\right): x, y \in X, x \neq y\right\}$ is a unital set of Jauch-Piron states).

It is well-known and easy to see that every state on a Boolean algebra is JauchPiron and that a unital set of states on a Boolean algebra is strongly order determining. Let us generalize the latter statement. 
3.4. Theorem. A set of Jauch-Piron states on an effect algebra with the maximality property is unital if and only if it is strongly order determining.

Proof. $\Leftarrow$ : See Lemma 2.3,

$\Rightarrow$ : Let $E$ be an effect algebra with the maximality property and with a unital set $S$ of Jauch-Piron states. Let $a, b \in E$ such that $a \not \leq b$. Let $c \in E$ be a maximal element of $[\mathbf{0}, a] \cap[\mathbf{0}, b]$. Then $c<a$ and therefore $a \ominus c \neq \mathbf{0}$. Since the set $S$ is unital, there is a state $s \in S$ such that $s(a \ominus c)=1$ and therefore $s(a)=1$. Let us suppose that $s(b)=1$ and seek a contradiction. Since $s$ Jauch-Piron, there is an element $d \in E$ such that $d \leq a \ominus c, d \leq b$ and $s(d)=1$. Hence $d \neq \mathbf{0}$ and $c<c \oplus d \leq a$. According to Proposition 3.3, $b$ is principal and therefore $c \oplus d \leq b$-this contradicts to the maximality of $c$.

Let us remark that Proposition 3.3 is a consequence of Theorem 3.4 and Proposition 2.5. Let us present examples that the assumptions in Theorem 3.4 cannot be omitted.

3.5. Example. Let $X=\{a, b, c, d\}, E$ be the family of even-element subsets of $X$ with the $\oplus$ operation defined as the union of disjoint sets. Then $(E, \oplus, \emptyset, X)$ is a finite (hence with the maximality property) concrete orthomodular poset and the set $S=\left\{s_{a}, s_{b}, s_{c}\right\}$ of states carried by points $a, b, c$ is a unital set of (two-valued) states on $E$ that is not strongly order determining: $\{a, d\} \not \leq\{a, b\}$ but there is no state $s \in S$ such that $s(\{a, d\})=1>s(\{a, b\})$. (States in $S$ are not Jauch-Piron.)

3.6. Example. Let $X_{1}, X_{2}, X_{3}, X_{4}$ be nonempty mutually disjoint sets, $X_{1}, X_{3}$ be infinite, $X=\bigcup_{i=1}^{4} X_{i}$,

$$
\begin{aligned}
E_{0} & =\left\{\emptyset, X_{1} \cup X_{2}, X_{2} \cup X_{3}, X_{3} \cup X_{4}, X_{4} \cup X_{1}, X\right\}, \\
E & =\left\{(A \backslash F) \cup(F \backslash A): F \subset X_{1} \cup X_{3} \text { is finite, } A \in E_{0}\right\},
\end{aligned}
$$

$A \oplus B=A \cup B$ for disjoint $A, B \in E$. Then $(E, \oplus, \emptyset, X)$ is a concrete orthomodular poset and the set $S=\left\{s_{x}: x \in X_{1} \cup X_{3}\right\}$ of states carried by points from $X_{1} \cup X_{3}$ is a unital set of (two-valued) Jauch-Piron states on $E$. The set $S$ is not strongly order determining because $X_{1} \cup X_{4} \not \leq X_{1} \cup X_{2}$ and for every $s \in S$ with $s\left(X_{1} \cup X_{4}\right)=1$ there is an $x \in X_{1}$ such that $s=s_{x}$ and therefore $s\left(X_{1} \cup X_{2}\right)=1$. ( $E$ does not have the maximality property.)

3.7. Theorem. Every Jauch-Piron effect algebra with a countable unital set of states is an orthomodular lattice.

Proof. Let $E$ be a Jauch-Piron effect algebra with a countable unital set $S$ of states. According to Lemma 3.2, $E$ has the maximality property. According to Theorem 3.4, the set $S$ is strongly order determining. According to Proposition 2.5. $E$ is an orthomodular poset. Let us show that $a \wedge b$ exists for every $a, b \in E$. (Then also $a \vee b=\left(a^{\prime} \wedge b^{\prime}\right)^{\prime}$ exists for every $a, b \in E$.) If $[\mathbf{0}, a] \cap[\mathbf{0}, b]=\{\mathbf{0}\}$ then $\mathbf{0}=a \wedge b$. Let us suppose that there is a nonzero element $c \in E$ such that $c \leq a, b$. Then there is a state $s \in S$ such that $s(c)=1$. Hence $s(a)=s(b)=1$ and the set $S_{a, b}=\{s \in S$ : $s(a)=s(b)=1\}$ is nonempty and countable. Let $s_{0}$ be a $\sigma$-convex combination (with nonzero coefficients) of all states from $S_{a, b}$. Then $s_{0}(a)=s_{0}(b)=1$. Since the state $s_{0}$ is Jauch-Piron, there is an element $c_{0} \in E$ such that $c_{0} \leq a, b$ and 
$s_{0}\left(c_{0}\right)=1$. Hence $s\left(c_{0}\right)=1$ for every $s \in S_{a, b}$. For every $c \in[\mathbf{0}, a] \cap[\mathbf{0}, b]$ and every $s \in S$ with $s(c)=1$ we have $s \in S_{a, b}$ and therefore $s\left(c_{0}\right)=1$. Since $S$ is strongly order determining, $c \leq c_{0}$ for every $c \in[\mathbf{0}, a] \cap[\mathbf{0}, b]$. Hence $c_{0}=a \wedge b$.

Let us present examples that the conditions in Theorem 3.7 cannot be omitted. There is a concrete (hence with a strongly order determining set of two-valued states) Jauch-Piron orthomodular poset that is not a lattice - see [5] (every unital set of states on it is uncountable). As the following example shows there is an orthomodular poset with a countable strongly order determining set of (two-valued) Jauch-Piron states that does not have the maximality property and therefore it is not a lattice (there are non-Jauch-Piron states).

3.8. Example. Let $X_{1}, X_{2}, X_{3}, X_{4}$ be mutually disjoint countable infinite sets, $X=$ $\bigcup_{i=1}^{4} X_{i}$

$$
\begin{aligned}
E_{0} & =\left\{\emptyset, X_{1} \cup X_{2}, X_{2} \cup X_{3}, X_{3} \cup X_{4}, X_{4} \cup X_{1}, X\right\}, \\
E & =\left\{(A \backslash F) \cup(F \backslash A): F \subset X \text { is finite, } A \in E_{0}\right\},
\end{aligned}
$$

$A \oplus B=A \cup B$ for disjoint $A, B \in E$. Then $(E, \oplus, \emptyset, X)$ is a concrete orthomodular poset and the set $S=\left\{s_{x}: x \in X\right\}$ of states carried by points is a countable strongly order determining set of two-valued Jauch-Piron states on $E$. The set $\left[\emptyset, X_{1} \cup X_{2}\right] \cap\left[\emptyset, X_{4} \cup X_{1}\right]$ consists of finite subsets of $X_{1}$, hence $E$ does not have the maximality property. As an example of a non-Jauch-Piron state we can take a $\sigma$-convex combination (with nonzero coefficients) of states from $\left\{s_{x}: x \in X_{1}\right\}$.

\section{Relationship of various conditions}

4.1. Theorem. Let $E$ be an effect algebra. Consider the following poperties:

(F) $E$ is finite.

(CF) $E$ is chain finite.

(OC) $E$ is orthocomplete.

(JPCU) $E$ is Jauch-Piron with a countable unital set of states.

(L) $E$ is a lattice.

(CU) For every $a, b \in E$, every chain in $[\mathbf{0}, a] \cap[\mathbf{0}, b]$ has an upper bound in $[\mathbf{0}, a] \cap[\mathbf{0}, b]$.

(M) $E$ has the maximality property.

Then the following implications hold: $(\mathrm{F}) \Rightarrow(\mathrm{CF}) \Rightarrow(\mathrm{OC}) \Rightarrow(\mathrm{CU}) \Rightarrow(\mathrm{M}),(\mathrm{JPCU}) \Rightarrow$ $(\mathrm{L}) \Rightarrow(\mathrm{CU})$.

Proof. $(\mathrm{F}) \Rightarrow(\mathrm{CF})$ : Obvious.

$(\mathrm{CF}) \Rightarrow(\mathrm{OC})$ : Every orthogonal system in a chain finite effect algebra is finite. Hence $E$ is orthocomplete.

$(\mathrm{OC}) \Rightarrow(\mathrm{CU})$ : Let $C$ be a chain in $[\mathbf{0}, a] \cap[\mathbf{0}, b]$. According to [4, Theorem 3.2], every chain in an orthocomplete effect algebra has a supremum. This supremum obviously belongs to $[\mathbf{0}, a] \cap[\mathbf{0}, b]$.

$(\mathrm{CU}) \Rightarrow(\mathrm{M})$ : Let $a, b \in E$. Since $[\mathbf{0}, a] \cap[\mathbf{0}, b] \supset\{\mathbf{0}\}$, the family of chains in $[\mathbf{0}, a] \cap[\mathbf{0}, b]$ is nonempty. According to Zorn's lemma, there is a maximal chain $C$ in 
$[\mathbf{0}, a] \cap[\mathbf{0}, b]$. According to the assumption, there is an upper bound $c \in[\mathbf{0}, a] \cap[\mathbf{0}, b]$ of $C$. Since the chain $C$ is maximal, $c \in C$ is a maximal element of $[\mathbf{0}, a] \cap[\mathbf{0}, b]$.

$(\mathrm{JPCU}) \Rightarrow(\mathrm{L})$ : See Theorem 3.7.

$(\mathrm{L}) \Rightarrow(\mathrm{CU})$ : Let $a, b \in E$. The element $a \wedge b$ is an upper bound for every chain in $[\mathbf{0}, a] \cap[\mathbf{0}, b]$.

Let us present examples that the scheme of implications in the previous theorem cannot be improved.

4.2. Example. Let $X$ be an infinite set, $y \notin X, E=\{\emptyset\} \cup\{\{x, y\}: x \in X\} \cup$ $\{X \backslash\{x\}: x \in X\} \cup\{X \cup\{y\}\}, A \oplus B=A \cup B$ for disjoint $A, B \in E$. Then $(E, \oplus, \emptyset, X \cup\{y\})$ is an infinite chain finite concrete orthomodular lattice.

4.3. Example. Let $X$ be an uncountable set, $E=\exp X$ with $A \oplus B=A \cup B$ for disjoint $A, B \in E$. Then $(E, \oplus, \emptyset, X)$ is an orthocomplete concrete orthomodular lattice (it forms a Boolean algebra) such that there is an uncountable set of mutually orthogonal elements. Hence it is not chain finite and every unital set of states on $E$ is uncountable.

4.4. Example. Let $X$ be a countable infinite set. Let $E$ be a family of finite and cofinite subsets of $X$ with the $\oplus$ operation defined as the union of disjoint sets. Then $(E, \oplus, \emptyset, X)$ is a concrete orthomodular lattice (it forms a Boolean algebra) fulfilling the condition (JPCU) (every state on a Boolean algebra is Jauch-Piron, there is a countable unital set of states carried by points) that is not orthocomplete.

4.5. Example. Let $X$ be a 6 -element set. Let $E$ be the family of even-element subsets of $X$ with the $\oplus$ operation defined as the union of disjoint sets from $E$. Then $(E, \oplus, \emptyset, X)$ is a finite concrete orthomodular poset that is not a latice.

4.6. Example. Let $X, Y$ be disjoint infinite countable sets,

$$
\begin{aligned}
E_{0} & =\{A \subset(X \cup Y): \operatorname{card}(A \cap X)=\operatorname{card}(A \cap Y) \text { is finite }\}, \\
E & =E_{0} \cup\left\{(X \cup Y) \backslash A: A \in E_{0}\right\},
\end{aligned}
$$

$A \oplus B=A \cup B$ for disjoint $A, B \in E$. Then $(E, \oplus, \emptyset, X \cup Y)$ is a concrete orthomodular poset with the maximality property. Let $X=\left\{x_{n}: n \in \mathbb{N}\right\}, y_{0} \in Y, f$ : $X \rightarrow Y \backslash\left\{y_{0}\right\}$ be a bijection, $A=(X \cup Y) \backslash\left\{x_{1}, f\left(x_{1}\right)\right\}, B=(X \cup Y) \backslash\left\{x_{1}, y_{0}\right\}$. Then the chain $\left\{\left\{x_{2}, \ldots, x_{n}, f\left(x_{2}\right), \ldots, f\left(x_{n}\right)\right\}: n \in \mathbb{N} \backslash\{1\}\right\}$ in $[\emptyset, A] \cap[\emptyset, B]$ does not have an upper bound in $[\emptyset, A] \cap[\emptyset, B]$, hence the condition (CU) from Theorem 4.1 is not fulfilled.

Let us remark that not all effect algebras have the maximality property (see Example 3.8).

\section{Acknowledgements}

The work was supported by the grant of the Grant Agency of the Czech Republic no. 201/07/1051 and by the research plan of the Ministry of Education of the Czech Republic no. 6840770010. 


\section{References}

[1] Dvurečenskij, A., Pulmannová, S.: New Trends in Quantum Structures. Kluwer Academic Publishers, Bratislava, 2000.

[2] Foulis, D. J., Bennett, M. K.: Effect algebras and unsharp quantum logics, Found. Phys. (1994) 24, 1331-1352.

[3] Foulis, D., Greechie, R., Rüttimann, G.: Filters and supports in orthoalgebras. Internat. J. Theoret. Phys. 31 (1992), 789-807.

[4] Jenča, G., Pulmannová, S.: Orthocomplete effect algebras. Proc. Amer. Math. Soc. 131 (2003), 2663-2671.

[5] Müller, V.: Jauch-Piron states on concrete quantum logics. Internat. J. Theoret. Phys. 32 (1993), 433-442.

[6] Tkadlec, J.: Central elements of effect algebras. Internat. J. Theoret. Phys. 43 (2004), 1363-1369.

[7] Tkadlec, J.: Conditions that force an orthomodular poset to be a Boolean algebra. Tatra Mt. Math. Publ. 10 (1997), 55-62. 